\title{
Porous aromatic framework (PAF-1) as hyperstable platform for enantioselective organocatalysis
}

\author{
Peng Chen ${ }^{1,3}$, Jin-Shi Sun ${ }^{1}$, Lei Zhang ${ }^{1}$, Wen-Yue Ma ${ }^{1}$, Fuxing Sun ${ }^{1}$ and Guangshan Zhu ${ }^{1,2 *}$
}

\begin{abstract}
High density of phenyl rings makes PAF-1 have robust structure and highly lipophilic pore, which make it very suitable for organocatalysis. However, there is no report about using PAF-1 as platform for enantioselective organocatalysis. In this paper, using PAF-1 as the platform, a chiral prolinamide catalytic site was introduced onto the framework of PAF-1 via a series of stepwise post-synthetic modifications, obtaining a novel PAF-supported chiral catalyst named PAF-1-NHPro. Then its enantioselective catalytic performance was studied by subjecting it to catalyze the model Aldol reaction between $p$-nitrobenzaldehyde and cyclohexanone. PAF-1-NHPro showed good diastereoselectivity and enantioselectivity with excellent and easy recyclability.
\end{abstract}

Keywords: porous aromatic frameworks, L-prolinamide, heterogeneous enantioselective organocatalysis, Aldol reaction

\section{INTRODUCTION}

Porous materials have been used for many practical applications that exploit the porosity of their structures. Among the applications, immobilization of homogeneous catalysts, especially chiral catalysts, on cavity surfaces of the solid porous materials is of great importance. In this field, the latest developments involved enantioselective catalysis based on metal-organic frameworks (MOFs) [114], covalent organic frameworks (COFs) [15-24] and porous organic polymers (POPs) [25-32]. Many advantages of MOFs, COFs and POPs for heterogeneous enantioselective catalysis have been elaborated by scientists. However metal-organic coordinative bonds in MOFs make the materials usually suffer from low stability to thermal treatments, water, and most organic solvents, which has restricted their further development in catalysis field. On the other hand, in the case of COFs, because the materials are all prepared by reversible reactions, the resultantly formed covalent bonds (most of them are boron-oxygen bonds or imine bonds) are also a hidden trouble for the stability of COFs under certain conditions and can interfere with some catalytic reactions, which might limit their application in enantioselective catalysis. Moreover, the POPs constru- cted only by stable covalent bonds are very stable but usually do not have robust structure and ordered pores. In recent years we have developed a series of porous aromatic frameworks (PAFs) featured by the high density of aromatic rings linked only by strong covalent carbon-carbon single bonds. PAFs can combine the advantages of MOFs, COFs and POPs and can be used for diverse applications [33-36]. Furthermore, it was found that PAF materials are perfect platforms for organocatalysis and organometallic catalysis by our group in recent studies [37,38].

Among the reported PAF materials, the most wellknown and intensively studied one is PAF-1 [39] developed by our group, which is the seminal work of PAFs and has many attractive and great prospects [34,40-42]. So far PAF-1 and functionalized PAF-1 have been widely used for adsorption [43-56], separation [57,58], heterogeneous catalysis [59], detection [60] and other diverse applications [61-64]. On the one hand, PAF-1 has high level of porosity and extraordinary stability to thermal treatments and almost all of the solvents. On the other hand, the framework structure of PAF-1 is so robust that the material can endure even very harsh reaction conditions, which makes PAF-1 very easily functionalized through established reactions. Although the above advantages have offered PAF-1 superior potentials as plat-

\footnotetext{
${ }^{1}$ State Key Laboratory of Inorganic Synthesis and Preparative Chemistry, College of Chemistry, Jilin University, Changchun 130012, China

${ }^{2}$ Key Laboratory of Polyoxometalate Science of the Ministry of Education, Faculty of Chemistry, Northeast Normal University, Changchun 130024, China

${ }^{3}$ Institute of Drug Discovery Technology, Ningbo University, Ningbo 315211, China

* Corresponding author (email: zhugs100@nenu.edu.cn)
} 
forms for enantioselective organocatalysis, this research field is still in its infant stage. To the best of our knowledge, in the enantioselective organo- catalysis field, the only related example is the use of defective PAF-1 as the platform for chiral organocatalyst, which was synthesized by one-pot copolymerization of the primitive tetrahedral building blocks and the low-connected functional chiral building blocks [32]. However, the introduction of the low-connected monomer could seriously influence the polymerization and generate a large number of defects, and thus the obtained defective framework was obviously different from that of PAF-1. In a word, PAF-1 has got less attention than they should in the enantioselective organocatalysis field given their vastly superior features. Hence, in this work we would pioneer the use of functionalized PAF-1 material in the enantioselective organocatalysis field.

As in the cases of the known solid catalysts, the basic but important issue is how to combine the PAF-1 framework and the chiral catalytically active site. In our design, the L-prolinamide was selected as the chiral catalytically active site. As we all know, L-proline and its derivatives are one type of the most famous organocatalysts, which can catalyze various enantioselective transformations including the Aldol, Michael, and Mannich reactions. Meanwhile, owing to the appropriate size as well as the easy preparation, the proline-type organocatalysts are one of the best candidates to investigate if a new solid material could be used as the platform for enantioselective catalysis. On the other hand, the Aldol reaction is an important reaction which is frequentlyused to investigate the catalytic activity of the catalyst supported by porous materials such as MOFs, COFs and POPs [1-9,23,24,32]. Thus in this paper we make an attempt to introduce the chiral catalytically active L-prolinamide unit into the PAF-1 material by stepwise post-synthetic modifications and investigate the catalytic performance of the obtained material for catalyzing the Aldol reaction.

\section{EXPERIMENTAL SECTION}

\section{General}

All moisture or oxygen-sensitive reactions were carried out under an argon atmosphere in oven or heat-dried flasks. The anhydrous solvents used were purified by distillation over the drying agents indicated in the square brackets and were transferred under argon: $\mathrm{N}, \mathrm{N}$-dimethylformamide (DMF) $\left[\mathrm{K}_{2} \mathrm{CO}_{3}\right]$, tetrahydrofuran (THF) $[\mathrm{Na}]$, chloroform $\left[\mathrm{CaCl}_{2}\right]$. All reactions were monitored by thin-layer chromatography (TLC) on gel $\mathrm{F}_{254}$ plates using UV light as visualizing agent (if applicable), and a solution of ammonium molybdate tetrahydrate $\left(50 \mathrm{~g} \mathrm{~L}^{-1}\right)$ in ethanol followed by heating as developing agents. The products were purified by flash column chromatography on silica gel (200-300 meshes) from the Qingdao Marine Chemical Factory in China.

${ }^{1} \mathrm{H}$ NMR spectra were recorded in $\mathrm{CDCl}_{3}$ solution on a Varian $300 \mathrm{MHz}$ instrument. Chemical shifts were denoted in $\mathrm{ppm}(\delta)$, and calibrated by using residual undeuterated solvent $\left(\mathrm{CHCl}_{3}(7.26 \mathrm{ppm})\right.$ or tetramethylsilane $(0.00 \mathrm{ppm}))$ as internal reference for ${ }^{1} \mathrm{H}$ NMR. Fourier transform infrared (FT-IR) spectra were recorded on a Bruker IFS 66v/S Fourier transform infrared spectrometer. The solid state ${ }^{13} \mathrm{C}$ CP/MAS TOSS (cross-polarization/magic-angle spinning total suppression of spinning sidebands) NMR spectrum was measured on Bruker Avance III WB 400 spectrometer with magic angle spinning at $8 \mathrm{kHz}$ frequency. Powder X-ray diffraction (PXRD) was performed by a Rigaku D/ MAX2550 diffractometer using $\mathrm{Cu}-\mathrm{Ka}$ radiation, $40 \mathrm{kV}$, $200 \mathrm{~mA}$ with a scanning rate of $1^{\circ} \mathrm{min}^{-1}(2 \theta)$. Thermogravimetric analysis (TGA) was performed using a Netzch Sta $449 \mathrm{c}$ thermal analyzer system at a heating rate of $10^{\circ} \mathrm{C} \mathrm{min}{ }^{-1}$ from room temperature to $800^{\circ} \mathrm{C}$ in an air atmosphere. The $\mathrm{N}_{2}$ adsorption-desorption isotherms were measured on a Quantachrome Autosorb-iQ2 analyzer. Elemental analyses were carried out on a vario EL cube elemental analyzer. Analytical HPLC was recorded on a HPLC machine equipped with Agilent 1100 series quaternary pump with a UV diode array detector. The chiral stationary phase was Daicel Chiralcel AD-H column.

\section{Synthesis of PAF-1}

Tetrakis(4-bromophenyl)methane $\quad(509 \mathrm{mg}, \quad 0.8 \mathrm{mmol})$ was added to a solution of 2,2'-bipyridyl (565 mg, $3.65 \mathrm{mmol})$, bis(1,5-cyclooctadiene)nickel $(0) \quad(1.0 \mathrm{~g}$, $3.65 \mathrm{mmol})$, and 1,5-cyclooctadiene $(0.45 \mathrm{~mL}$, $3.65 \mathrm{mmol})$ in anhydrous DMF/THF $(60 \mathrm{~mL} / 90 \mathrm{~mL})$, and the mixture was stirred for $60 \mathrm{~h}$ at room temperature under nitrogen atmosphere. Then concentrated hydrochloric acid $(60 \mathrm{~mL})$ was added slowly, and the resulting mixture was stirred for $12 \mathrm{~h}$. The precipitate was collected by filtration, then washed with $2 \mathrm{~mol} \mathrm{~L}^{-1}$ hydrochloric acid $(1 \times 100 \mathrm{~mL}), \quad 1 \mathrm{~mol} \mathrm{L^{-1 }}$ hydrochloric acid $(2 \times$ $100 \mathrm{~mL})$, water $(4 \times 100 \mathrm{~mL})$ and methanol $(4 \times 100 \mathrm{~mL})$, and dried in vacuum at $150^{\circ} \mathrm{C}$ for $12 \mathrm{~h}$ to produce PAF-1 (248 mg, 98\% yield). Elemental analysis calcd. (\%) for $\mathrm{C}_{25} \mathrm{H}_{16}$ : C 94.90, H 5.10; found: C 94.73, H 5.27. 


\section{Synthesis of PAF-1-NO}

To a suspension of PAF-1 $(240 \mathrm{mg})$ in $\mathrm{Ac}_{2} \mathrm{O}(120 \mathrm{~mL})$ in an ice bath, $4.8 \mathrm{~mL}$ fuming nitric acid was gradually added. The resultant reaction mixture was then stirred at room temperature for 4 days. Subsequently, the mixture was poured into a large amount of water, and the solid was filtrated, washed with water $(5 \times 100 \mathrm{~mL})$ substantially, and then dried in vacuum at $120^{\circ} \mathrm{C}$ for $12 \mathrm{~h}$ to give PAF-1- $\mathrm{NO}_{2}(293 \mathrm{mg})$. Elemental analysis (\%) found: C 73.41, H 3.99, N 6.35.

\section{Synthesis of PAF-1-NH}

$250 \mathrm{mg}$ PAF- $1-\mathrm{NO}_{2}$ and $8.2 \mathrm{~g} \mathrm{SnCl}_{2} \cdot 2 \mathrm{H}_{2} \mathrm{O}$ were suspended in $50 \mathrm{~mL}$ ethanol. The resultant mixture was stirred at $70^{\circ} \mathrm{C}$ for $12 \mathrm{~h}$. The solid was filtrated and suspended in $50 \mathrm{~mL}$ concentrated hydrochloric acid. Then the mixture was centrifuged and the obtained solid was washed with water $(3 \times 100 \mathrm{~mL})$ and ethanol $(3 \times 100 \mathrm{~mL})$. The product was dried in vacuum at $120^{\circ} \mathrm{C}$ for $12 \mathrm{~h}$ to produce PAF-1- $\mathrm{NH}_{2}(211 \mathrm{mg})$. Elemental analysis (\%) found: C 85.23, H 5.69, N 7.38.

\section{Synthesis of L-proline acid chloride hydrochloride (L-Pro- $\mathrm{Cl} \cdot \mathrm{HCl}$ )}

This compound was synthesized according to the modified literature step [65]. To a suspension of phosphorus pentachloride $(38.0 \mathrm{~g}, 182.5 \mathrm{mmol})$ in chloroform $(100 \mathrm{~mL})$ was slowly added L-proline $(20.0 \mathrm{~g}$, $173.7 \mathrm{mmol}$ ) in small portions under argon, keeping the reaction temperature below $10^{\circ} \mathrm{C}$. The resultant reaction mixture was stirred for $30 \mathrm{~min}$ below $10^{\circ} \mathrm{C}$. Then the solid was filtered under argon, washed with chloroform $(1 \times 20 \mathrm{~mL})$ and dried in vacuum at room temperature, affording L-Pro-Cl-HCl (23.05 g, $135.6 \mathrm{mmol}$, 78\% yield) as a white solid.

\section{Synthesis of PAF-1-NHPro}

PAF-1- $\mathrm{NH}_{2}(150 \mathrm{mg})$ obtained above was added anhydrous THF $(50 \mathrm{~mL})$ and L-Pro-Cl. $\mathrm{HCl}(1.09 \mathrm{~g})$. Then the resulting mixture was stirred for one day at room temperature. Then $50 \mathrm{~mL}$ saturated $\mathrm{Na}_{2} \mathrm{CO}_{3}$ aqueous solution was added to the reaction mixture. The resulting mixture was filtrated. The resultant solid was washed with $\mathrm{H}_{2} \mathrm{O}$ $(4 \times 50 \mathrm{~mL})$, THF $(4 \times 50 \mathrm{~mL})$ and $\mathrm{CH}_{2} \mathrm{Cl}_{2}(4 \times 50 \mathrm{~mL})$, dried in vacuum at $120^{\circ} \mathrm{C}$ for $12 \mathrm{~h}$, yielding the desired PAF-1NHPro (175 mg). Elemental analysis (\%) found: C 75.88, H 6.26, N 8.83.

\section{Typical procedure for Aldol reaction catalyzed by PAF-1-} NHPro

To a mixture of $p$-nitrobenzaldehyde $(0.25 \mathrm{mmol})$, PAF-
1-NHPro $(27 \mathrm{mg})$, glacial acetic acid (HOAc) $(0.2 \mathrm{mmol})$ and $m$-xylene $(0.5 \mathrm{~mL})$ was added cyclohexanone $(2.5 \mathrm{mmol})$ at $-20^{\circ} \mathrm{C}$. The resulting mixture was stirred at $-20^{\circ} \mathrm{C}$ for 7 days. Then the mixture was centrifuged and the obtained solid was washed with THF $(6 \times 5 \mathrm{~mL})$. The combined organic solutions were evaporated to dryness. The resultant residue was directly column chromatographed over silica gel (200-300 mesh) to afford the mixed Aldol product. The diastereomeric ratio (dr) was determined by ${ }^{1} \mathrm{H}-\mathrm{NMR}$ analysis of the mixed Aldol product. ${ }^{1} \mathrm{H}$ NMR $\left(300 \mathrm{MHz}, \mathrm{CDCl}_{3}\right): \delta=8.23-8.14(\mathrm{~m}$, $2 \mathrm{H}$ for anti isomer and $2 \mathrm{H}$ for syn isomer), 7.53-7.44 (m, $2 \mathrm{H}$ for anti isomer and $2 \mathrm{H}$ for syn isomer), 5.47 (s, $1 \mathrm{H}$ for syn isomer), 4.89 (d, $J=8.4 \mathrm{~Hz}, 1 \mathrm{H}$ for anti isomer), 2.65 -2.27 ( $\mathrm{m}, 3 \mathrm{H}$ for anti isomer and $3 \mathrm{H}$ for syn isomer), 2.16 $-2.01(\mathrm{~m}, 1 \mathrm{H}$ for anti isomer and $1 \mathrm{H}$ for syn isomer), 1.90-1.22 ppm (m, $5 \mathrm{H}$ for anti isomer and $5 \mathrm{H}$ for syn isomer). The enantiomeric excess (ee) was determined by HPLC analysis with a Daicel Chiralcel AD-H column [hexane/2-propanol=90:10, flow rate $1 \mathrm{~mL} \mathrm{~min}^{-1}, t_{\mathrm{R}}$ (anti isomer) $=22.6 \mathrm{~min}$ (minor), $30.5 \mathrm{~min}$ (major)]. The catalyst was directly dried in vacuum at $40^{\circ} \mathrm{C}$ for $12 \mathrm{~h}$ for reuse when required.

\section{RESULTS AND DISCUSSION}

Our strategy for introducing chiral catalytically active site to the PAF-1 is via a series of stepwise post-synthetic modifications. Inspired by the excellent work of $\mathrm{Ma}$ and co-workers [59], in which PAF-1 was bifunctionalized as a platform for cascade catalysis, we used a similar method to graft the chiral L-prolinamide functional group onto the framework of PAF-1. As shown in Scheme 1, PAF-1 was nitrated by $\mathrm{HNO}_{3}$ and then reduced by $\mathrm{SnCl}_{2}$ to afford PAF-1- $\mathrm{NH}_{2}$, which further reacted with L-proline acid chloride hydrochloride (L-Pro-Cl-HCl) to afford PAF-1-NHPro.

First, FT-IR spectra of the materials, as shown in Fig. 1, were used to verify the preparation of the chiral catalytically active site tethered PAF by using the above strategy. Compared with PAF-1, the FT-IR spectrum of PAF-1- $\mathrm{NO}_{2}$ shows two strong peaks at 1,533 and $1,350 \mathrm{~cm}^{-1}$ which are the characteristic peaks of $-\mathrm{NO}_{2}$ group, indicating the introduction of nitro groups into the PAF-1. After reduction process, in the FT-IR spectrum of PAF-1- $\mathrm{NH}_{2}$ the above two peaks of $-\mathrm{NO}_{2}$ group disappeared and the characteristic double peaks of $-\mathrm{NH}_{2}$ $\left(3,473\right.$ and $\left.3,383 \mathrm{~cm}^{-1}\right)$ appeared, together indicating the construction of the designed amino-containing PAF material PAF-1-NH $\mathrm{NH}_{2}$. In the FT-IR spectrum of PAF-1NHPro, the strong attenuation of the characteristic dou- 

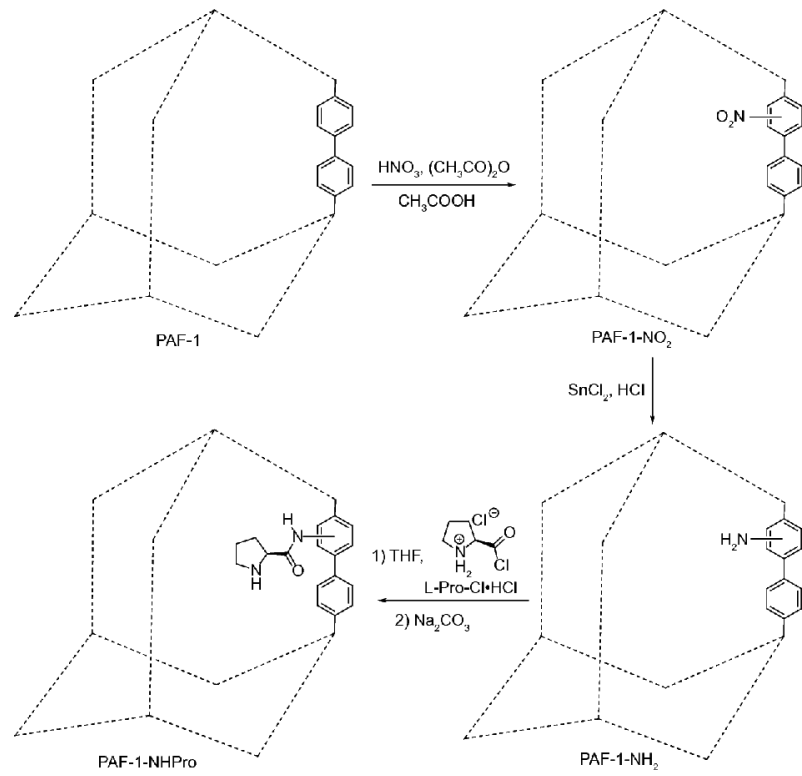

Scheme 1 Synthetic route to L-prolinamide tethered PAF (PAF-1NHPro).

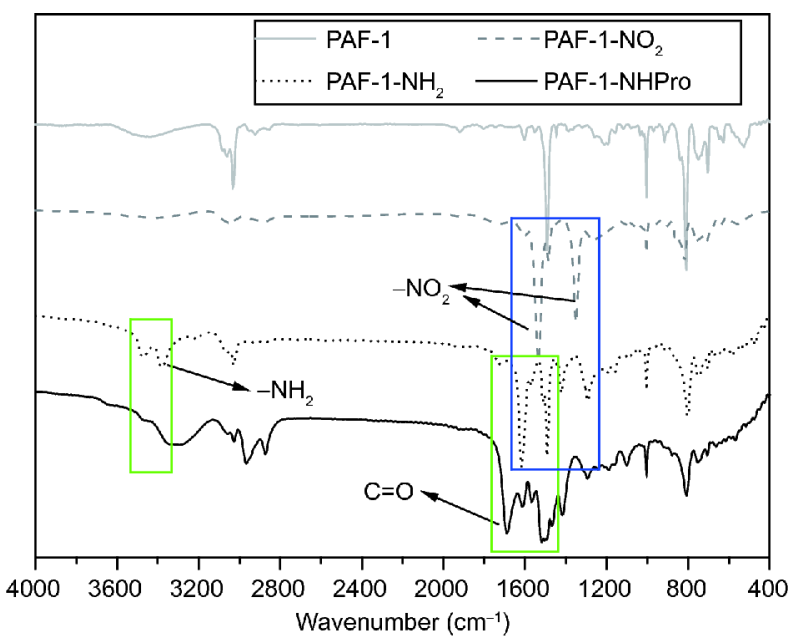

Figure 1 FT-IR spectra of PAF-1, PAF-1-NO $\mathrm{NO}_{2}$ PAF-1-NH $\mathrm{N}_{2}$ and PAF-1NHPro.

ble peaks of $-\mathrm{NH}_{2}$ and the appearance of the characteristic peaks of carbonyl group (the new peaks at around $1,600 \mathrm{~cm}^{-1}$ ) indicated that the L-prolinamide group was introduced into the PAF material.

The chemical composition of PAF-1-NHPro was further characterized by solid-state ${ }^{13} \mathrm{C}$ CP/MAS TOSS NMR spectroscopy (Fig. 2). The solid-state ${ }^{13} \mathrm{C}$ NMR (Fig. 2) of PAF-1-NHPro showed one broad peak around $170 \mathrm{ppm}$ which was the characteristic peak of the amide carbon. The four peaks at 24, 29, 46, $60 \mathrm{ppm}$ could be assigned to the aliphatic carbons in the proline-type ring. The peak

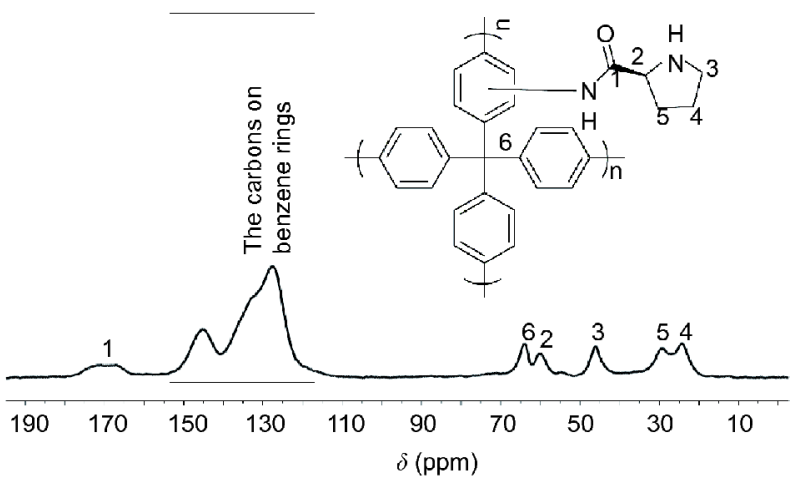

Figure 2 Solid state ${ }^{13} \mathrm{C}$ CP/MAS TOSS NMR Spectrum of PAF-1NHPro. The assignments of the ${ }^{13} \mathrm{C}$ NMR signals are indicated (top).

corresponding to the aliphatic quaternary carbon linked by four benzene rings was observed at $64 \mathrm{ppm}$ and the peaks corresponding to the aromatic carbons were observed at $117-152 \mathrm{ppm}$ in the spectrum. In a word, the peaks in the ${ }^{13} \mathrm{C}$ NMR spectrum agree well with the designed immobilized catalyst, which confirms that the Lprolinamide catalyst has been well embedded into the PAF material.

The above analyses of the FT-IR spectra of the four PAF materials and the solid-state ${ }^{13} \mathrm{C}$ NMR spectrum of PAF-1-NHPro together demonstrated that PAF-1-NHPro with L-prolinamide catalytic unit was obtained as designed. In addition, the PXRD patterns (Fig. S1) of PAF-1, PAF-1-NO $\mathrm{N}_{2}$ PAF-1-NH $\mathrm{N}_{2}$ and PAF-1-NHPro indicated that the four PAF materials all do not have longrange ordered structures.

The nitrogen adsorption-desorption isotherms of PAF-1, PAF-1-NO $\mathrm{N}_{2}$, PAF-1-NH $\mathrm{N}_{2}$ and PAF-1-NHPro (Fig. 3) all showed a rapid uptake at low relative pressure,

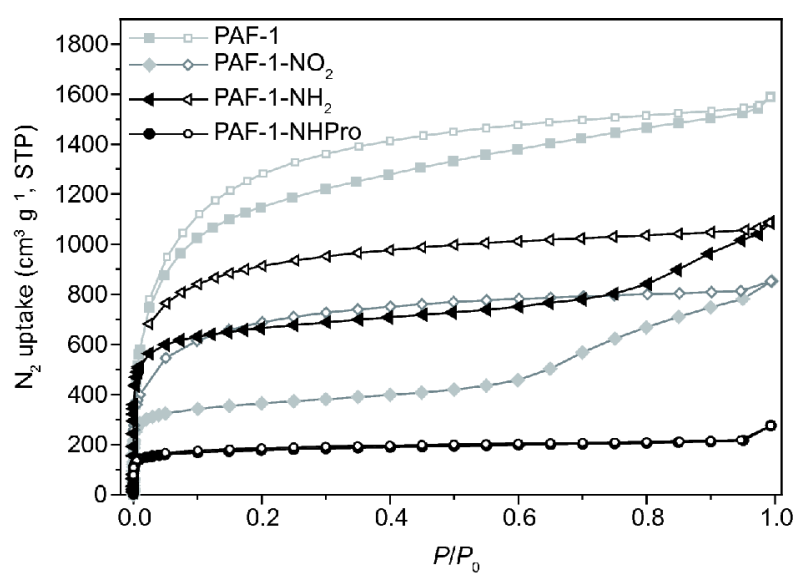

Figure 3 Nitrogen adsorption (solid symbols)-desorption (open symbols) isotherms of PAF-1, PAF-1-NO $\mathrm{N}_{2}$ PAF-1-NH $\mathrm{NH}_{2}$ and PAF-1-NHPro measured at $77 \mathrm{~K}$. 
which indicated the existence of micropores in these materials. The Brunauer-Emmett-Teller (BET) surface area was $4,358 \mathrm{~m}^{2} \mathrm{~g}^{-1}$ for PAF-1, $1,358 \mathrm{~m}^{2} \mathrm{~g}^{-1}$ for PAF-1$\mathrm{NO}_{2}, 2,542 \mathrm{~m}^{2} \mathrm{~g}^{-1}$ for PAF-1-NH${ }_{2}$ and $677 \mathrm{~m}^{2} \mathrm{~g}^{-1}$ for PAF-1-NHPro. The BET surface area changes from PAF-1 to PAF-1-NO $\mathrm{NO}_{2}$ to PAF-1-NH $\mathrm{NH}_{2}$ were in good agreement with the intrinsic size properties of the corresponding functional groups. Noteworthily, there is a sharp decrease of the BET surface area from $2,542 \mathrm{~m}^{2} \mathrm{~g}^{-1}$ for PAF-1- $\mathrm{NH}_{2}$ to $677 \mathrm{~m}^{2} \mathrm{~g}^{-1}$ for PAF-1-NHPro. This sharp decrease further indicated that the chiral L-prolinamide units were introduced into the pores of PAF-1$\mathrm{NH}_{2}$ and thus PAF-1-NHPro was synthesized as expected.

Thermal stability of the obtained PAF materials was tested by TGA. As shown in Fig. 4, PAF-1, PAF-1- $\mathrm{NO}_{2}$ and PAF-1- $\mathrm{NH}_{2}$ all showed almost no weight loss below $350^{\circ} \mathrm{C}$, suggesting their high thermal stability. The obvious decomposition of the framework started at about $400^{\circ} \mathrm{C}$ for PAF- 1 and PAF-1- $\mathrm{NH}_{2}$ and at about $350^{\circ} \mathrm{C}$ for PAF-1- $\mathrm{NO}_{2}$. When the temperature reached $560^{\circ} \mathrm{C}$ for PAF-1- $\mathrm{NO}_{2}$ and $620^{\circ} \mathrm{C}$ for PAF- 1 and PAF-1- $\mathrm{NH}_{2}$ the complete decomposition of the materials was investigated. Interestingly, after introducing the L-prolinamide units, repeated experiments ensured that PAF-1NHPro (black solid curve, Fig. 4) showed a $4 \%$ weight loss before $100^{\circ} \mathrm{C}$, which might be due to the gradual disappearance of some guest molecules in the pores of the PAF material. Unlike other three PAF materials, this distinctive phenomenon of PAF-1-NHPro indicates that the prolinamide unit has stronger interaction force (such as intermolecular hydrogen bonding) with the guest molecule which most likely is water. In addition, PAF-1NHPro showed a slow $30 \%$ weight loss between

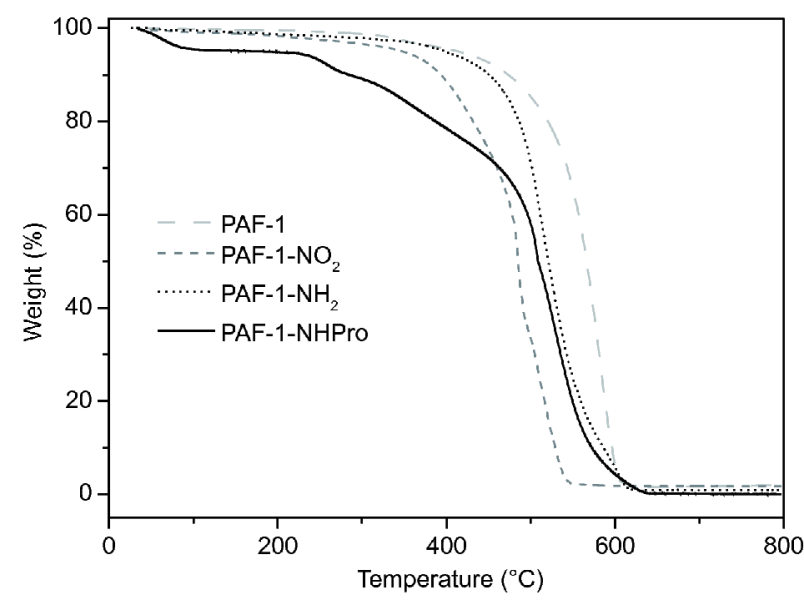

Figure 4 TGA plots of PAF-1, PAF-1-NO $\mathrm{N}_{2}, \mathrm{PAF}-1-\mathrm{NH}_{2}$ and PAF-1NHPro. $230-480^{\circ} \mathrm{C}$, which was attributed to the decomposition of the L-prolinamide unit. Further obvious decomposition of the PAF-1-NHPro framework started at $480^{\circ} \mathrm{C}$ and the material completely decomposed when the temperature reached $630^{\circ} \mathrm{C}$. Although PAF-1-NHPro showed a lower thermal stability which mainly resulted from the intrinsic property of the catalytic unit, the decomposition temperature $\left(230^{\circ} \mathrm{C}\right)$ is high enough to meet the need of most enantioselective organocatalysis. Furthermore, PAF-1NHPro could not be dissolved or decomposed in almost all common solvents such as THF, $\mathrm{CH}_{2} \mathrm{Cl}_{2}, \mathrm{CHCl}_{3}$, EtOAc, toluene, methanol $(\mathrm{MeOH})$, ethanol $(\mathrm{EtOH})$, DMSO, dimethylformamide (DMF), xylene and water, which makes it very suitable for heterogeneous organocatalysis.

To conclude, the above characterization of the obtained materials clearly proved the successful preparation of PAF-1-NHPro with chiral catalytically active site as designed.

With the expected PAF-1-NHPro in hand, its catalytic performance was evaluated, adopting Aldol reaction between $p$-nitrobenzaldehyde and cyclohexanone as a model reaction. Because the quantity of the immobilized catalytic site was difficult to accurately calculate for PAF1-NHPro, the catalyst loading was selected by an easy initial investigation and then remained unchanged in the control experiments as indicated in Table 1 . This is different from the research on the homogeneous catalysis. First, we screened various solvents (neat, hexane, $\mathrm{Et}_{2} \mathrm{O}$, $m$-xylene) and $m$-xylene gave the best results in terms of diastereoselectivity, enantioselectivity and yield of the current catalytic reaction (entry 1-4, Table 1 ). So we used $m$-xylene as the solvent to further investigate our heterogeneous catalysis. The elevation of reaction temperature from $-20^{\circ} \mathrm{C}$ to room temperature increased the reactivity of the current reaction but seriously reduced the enantioselectivity and diastereoselectivity (entry 5 , Table 1 ). In addition, the material PAF-1- $\mathrm{NH}_{2}$ (entry 6, Table 1) could not catalyze the current reaction under the optimized conditions, indicating that the introduced chiral prolinamide unit in PAF-1-NHPro is indeed the effective catalytic site for the current reaction. The supernatant liquid of the $m$-xylene suspension of PAF-1-NHPro had no catalytic activity (entry 7 , Table 1), which definitely indicated no leakage of catalytically active species from the PAF-1-NHPro catalyst during the catalysis process. It is noteworthy that the operation after reaction is very simple, in which only centrifugation is needed to remove the solid catalyst and the obtained solution could be further directly purified by evaporation and flash column 
Table 1 The control experiments for PAF-1-NHPro catalyzed Aldol reaction ${ }^{\mathrm{a}}$

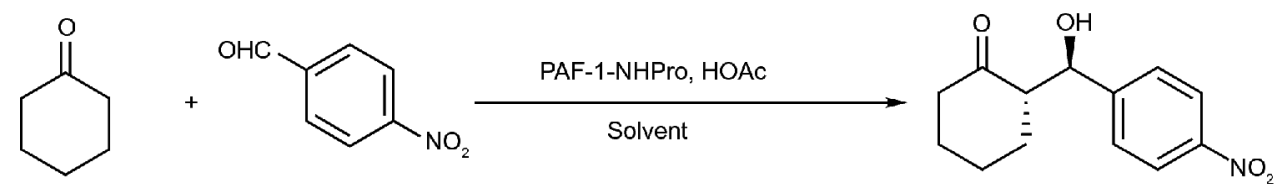

\begin{tabular}{|c|c|c|c|c|c|c|c|}
\hline Entry & Catalyst & Solvents & $T\left({ }^{\circ} \mathrm{C}\right)$ & Time & Yield $(\%)^{\mathrm{b}}$ & $\mathrm{dr}(\operatorname{anti} / \mathrm{syn})^{\mathrm{c}}$ & ee (anti) $(\%)^{\mathrm{d}}$ \\
\hline 1 & PAF-1-NHPro & Neat & -20 & 4 days & 80 & $5.0: 1$ & 41 \\
\hline 2 & PAF-1-NHPro & Hexane & -20 & 7 days & 41 & $9.6: 1$ & 68 \\
\hline 3 & PAF-1-NHPro & $\mathrm{Et}_{2} \mathrm{O}$ & -20 & 7 days & 59 & $5.1: 1$ & 51 \\
\hline 4 & PAF-1-NHPro & $m$-Xylene & -20 & 7 days & 70 & 10.3:1 & 71 \\
\hline 5 & PAF-1-NHPro & $m$-Xylene & RT & 1.5 days & 95 & $2.8: 1$ & 43 \\
\hline 6 & PAF-1-NH & $m$-Xylene & -20 & 7 days & 0 & I & I \\
\hline $7^{\mathrm{e}}$ & $\begin{array}{l}\text { The supernatant liquid of } \\
\text { the } m \text {-xylene suspension } \\
\text { of PAF-1-NHPro }\end{array}$ & I & -20 & 7 days & 0 & I & I \\
\hline
\end{tabular}

a) Conditions: a mixture of $p$-nitrobenzaldehyde $(0.25 \mathrm{mmol})$, catalyst $(27 \mathrm{mg})$, HOAc $(0.2 \mathrm{mmol})$ and cyclohexanone $(2.5 \mathrm{mmol})$ in solvents $(0.5 \mathrm{~mL})$ was stirred at the indicated temperature for the indicated time. b) The isolated yield of the mixture of the anti and syn isomers. c) Determined by ${ }^{1} \mathrm{H}$ NMR. d) Determined by chiral HPLC. e) $54 \mathrm{mg}$ PAF-1-NHPro was immersed in $1 \mathrm{~mL}$ of $m$-xylene for 7 days at $-20^{\circ} \mathrm{C}$, after centrifugation. The supernatant liquid $(0.5 \mathrm{~mL})$ was added to cyclohexanone $(2.5 \mathrm{mmol}), p$-nitrobenzaldehyde $(0.25 \mathrm{mmol})$ and $\mathrm{HOAc}(0.2 \mathrm{mmol})$, and then the resulting mixture was stirred at $-20^{\circ} \mathrm{C}$ for 7 days.

chromatography to yield the desired product.

Under our optimized conditions, the recyclability that is an important factor for a heterogeneous catalyst was tested. As shown in Table 2, PAF-1-NHPro was subjected to 10 cycles of the Aldol reaction between $p$-nitrobenzaldehyde and cyclohexanone. In each cycle, the reaction was driven to react for the same time. After each cycle, the catalyst could be easily separated from the reaction system by centrifugation followed by washing with THF. The recovered catalyst was dried and could be directly reused in the next cycle. It was demonstrated that in the 10 cycles, there was no observable loss of the $\mathrm{dr}$ and ee value of the catalytic reaction. The reaction yields had a slightly decline in the 10 cycles, which is mainly due to the material loss in the catalyst recovering process. To further investigate the stability of PAF-1-NHPro in the recycle test for the Aldol reaction, the recycled PAF-1NHPro after 10 cycles was characterized by FT-IR spectrum and nitrogen adsorption-desorption isotherms. Besides some weak peaks that might be attributed to a very small amount of adsorbed Aldol product or byproduct on the recycled catalyst, the FT-IR spectra (Fig. S2) of the fresh PAF-1-NHPro and the recycled catalyst after 10 cycles were almost the same. As shown in the nitrogen adsorption-desorption isotherms (Fig. S3), compared with the fresh PAF-1-NHPro, the recycled catalyst after 10 cycles showed only a slight decrease of the BET surface area (from $677 \mathrm{~m}^{2} \mathrm{~g}^{-1}$ for the fresh PAF-1-NHPro to $644 \mathrm{~m}^{2} \mathrm{~g}^{-1}$ for the recycled PAF-1NHPro). The above results indicated that the functional groups together with the framework and pores of PAF-1NHPro remained almost unchanged after 10 cycles of the Aldol reaction. Notably, PAF-1-NHPro could be kept under air at ambient temperature for 30 days and showed no loss of activity. The above results clearly proved the exceptional chemical stability and perfect recyclability of the catalyst PAF-1-NHPro.

\section{CONCLUSIONS}

In summary, we have developed a route to a PAF-1 based material (PAF-1-NHPro) with chiral catalytically active site during stepwise post-synthetic modifications. PAF-1NHPro exhibited good diastereoselectivity and enantioselectivity for catalyzing the Aldol reaction between cyclohexanone and p-nitrobenzaldehyde. More importantly, PAF-1-NHPro demonstrated exceptional chemical stability and perfect recyclability, that is, it could undergo at least 10 cycles without any loss of diastereoselectivity and enantioselectivity in the current catalysis system. Our work demonstrated that the PAF materials are promising candidates as a new solid platform for efficient green enantioselective organocatalysis. In parti- 
Table 2 Recovery and reuse of PAF-1-NHPro in the Aldol reaction ${ }^{\mathrm{a}}$

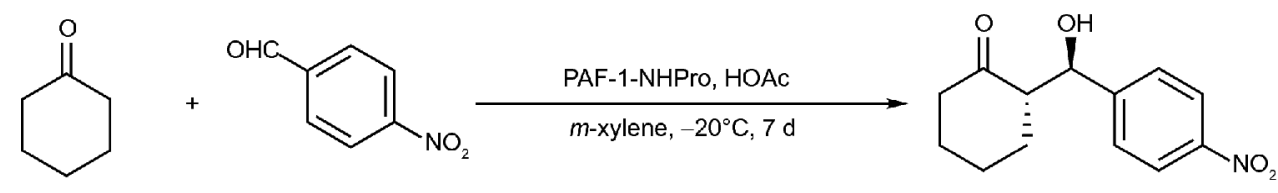

\begin{tabular}{cccc}
\hline Cycle & ${\text { Yield }(\%)^{\mathrm{b}}}^{\mathrm{dr}(\mathrm{anti} / \mathrm{syn})^{\mathrm{c}}}$ & ee (anti) $(\%)^{\mathrm{d}}$ \\
\hline 1 & 68 & $10.2: 1$ & 68 \\
2 & 71 & $10.5: 1$ & 71 \\
4 & 68 & $10.6: 1$ & 68 \\
6 & 66 & $10.8: 1$ & 70 \\
8 & 60 & $10.8: 1$ & 75 \\
10 & 54 & $10.6: 1$ & 73 \\
\hline
\end{tabular}

a) Conditions: a mixture of $p$-nitrobenzaldehyde $(0.25 \mathrm{mmol})$, PAF-1-NHPro $(27 \mathrm{mg})$, HOAc $(0.2 \mathrm{mmol})$ and cyclohexanone $(2.5 \mathrm{mmol}) \mathrm{in} \mathrm{m}$ xylene $(0.5 \mathrm{~mL})$ was stirred at $-20^{\circ} \mathrm{C}$ for $7 \mathrm{~d}$. After centrifugation and washing with THF $(6 \times 5 \mathrm{~mL})$ and drying in vacuum at $40^{\circ} \mathrm{C}$ for $12 \mathrm{~h}$, the catalyst was directly reused in the next cycle. b) The isolated yield of the mixture of the anti and syn isomers. c) Determined by ${ }^{1} \mathrm{H}$ NMR. d) Determined by chiral HPLC.

cular, the perfect and easy recyclability of PAF-1-NHPro has provided this new type of heterogeneous catalyst high potentials for large-scale industrial production of chiral chemical products in chemical industry and pharmaceutical industry. Therefore, the presented work opens an attractive door for PAFs as solid supported materials for the chiral organocatalysts. Predictably, a variety of chiral catalytic units can be incorporated into the pores of various PAFs and the resulting chiral PAF supported catalysts may catalyze diverse types of asymmetric transformations. Further work along this line is in progress.

Received 9 May 2018; accepted 28 June 2018; published online 10 August 2018

1 Banerjee M, Das S, Yoon M, et al. Postsynthetic modification switches an achiral framework to catalytically active homochiral metal-organic porous materials. J Am Chem Soc, 2009, 131: 75247525

2 Dang D, Wu P, He C, et al. Homochiral metal-organic frameworks for heterogeneous asymmetric catalysis. J Am Chem Soc, 2010, 132: 14321-14323

3 Lun DJ, Waterhouse GIN, Telfer SG. A general thermolabile protecting group strategy for organocatalytic metal-organic frameworks. J Am Chem Soc, 2011, 133: 5806-5809

4 Zhu W, He C, Wu P, et al. "Click" post-synthetic modification of metal-organic frameworks with chiral functional adduct for heterogeneous asymmetric catalysis. Dalton Trans, 2012, 41: 30723077

5 Lili L, Xin Z, Shumin R, et al. Catalysis by metal-organic frameworks: proline and gold functionalized MOFs for the aldol and three-component coupling reactions. RSC Adv, 2014, 4: 1309313107
6 Liu Y, Xi X, Ye C, et al. Chiral metal-organic frameworks bearing free carboxylic acids for organocatalyst encapsulation. Angew Chem Int Ed, 2014, 53: 13821-13825

7 Bonnefoy J, Legrand A, Quadrelli EA, et al. Enantiopure peptidefunctionalized metal-organic frameworks. J Am Chem Soc, 2015, 137: 9409-9416

8 Kutzscher C, Hoffmann HC, Krause S, et al. Proline functionalization of the mesoporous metal-organic framework DUT-32. Inorg Chem, 2015, 54: 1003-1009

9 Kutzscher C, Nickerl G, Senkovska I, et al. Proline functionalized UiO-67 and UiO-68 type metal-organic frameworks showing reversed diastereoselectivity in aldol addition reactions. Chem Mater, 2016, 28: 2573-2580

10 Ma L, Abney C, Lin W. Enantioselective catalysis with homochiral metal-organic frameworks. Chem Soc Rev, 2009, 38: 1248-1256

11 Seo JS, Whang D, Lee $\mathrm{H}$, et al. A homochiral metal-organic porous material for enantioselective separation and catalysis. Nature, 2000, 404: 982-986

12 Yoon M, Srirambalaji R, Kim K. Homochiral metal-organic frameworks for asymmetric heterogeneous catalysis. Chem Rev, 2012, 112: 1196-1231

13 Leus K, Liu YY, Van Der Voort P. Metal-organic frameworks as selective or chiral oxidation catalysts. Catal Rev, 2014, 56: 1-56

14 Bhattacharjee S, Khan M, Li X, et al. Recent progress in asymmetric catalysis and chromatographic separation by chiral metalorganic frameworks. Catalysts, 2018, 8: 120

15 Ding SY, Wang W. Covalent organic frameworks (COFs): from design to applications. Chem Soc Rev, 2013, 42: 548-568

$16 \mathrm{Xu} \mathrm{H}$, Chen X, Gao J, et al. Catalytic covalent organic frameworks via pore surface engineering. Chem Commun, 2014, 50: 1292-1294

$17 \mathrm{Xu} \mathrm{H}$, Gao J, Jiang D. Stable, crystalline, porous, covalent organic frameworks as a platform for chiral organocatalysts. Nat Chem, 2015, 7: 905-912

18 Wang X, Han X, Zhang J, et al. Homochiral 2D porous covalent organic frameworks for heterogeneous asymmetric catalysis. J Am Chem Soc, 2016, 138: 12332-12335 
19 Ma HC, Kan JL, Chen GJ, et al. Pd NPs-loaded homochiral covalent organic framework for heterogeneous asymmetric catalysis. Chem Mater, 2017, 29: 6518-6524

20 Han X, Xia Q, Huang J, et al. Chiral covalent organic frameworks with high chemical stability for heterogeneous asymmetric catalysis. J Am Chem Soc, 2017, 139: 8693-8697

21 Liu G, Sheng J, Zhao Y. Chiral covalent organic frameworks for asymmetric catalysis and chiral separation. Sci China Chem, 2017, 60: 1015-1022

22 Han X, Zhang J, Huang J, et al. Chiral induction in covalent organic frameworks. Nat Commun, 2018, 9: 1294

23 Xu HS, Ding SY, An WK, et al. Constructing crystalline covalent organic frameworks from chiral building blocks. J Am Chem Soc, 2016, 138: 11489-11492

24 Zhang J, Han X, Wu X, et al. Multivariate chiral covalent organic frameworks with controlled crystallinity and stability for asymmetric catalysis. J Am Chem Soc, 2017, 139: 8277-8285

25 Wang CA, Zhang ZK, Yue T, et al. "Bottom-up" embedding of the Jørgensen-Hayashi catalyst into a chiral porous polymer for highly efficient heterogeneous asymmetric organocatalysis. Chem Eur J, 2012, 18: 6718-6723

26 An WK, Han MY, Wang CA, et al. Insights into the asymmetric heterogeneous catalysis in porous organic polymers: constructing a taddol-embedded chiral catalyst for studying the structure-activity relationship. Chem Eur J, 2014, 20: 11019-11028

27 Dong J, Liu Y, Cui Y. Chiral porous organic frameworks for asymmetric heterogeneous catalysis and gas chromatographic separation. Chem Commun, 2014, 50: 14949-14952

28 Zhang Y, Ying JY. Main-chain organic frameworks with advanced catalytic functionalities. ACS Catal, 2015, 5: 2681-2691

29 Sun Q, Dai Z, Meng X, et al. Homochiral porous framework as a platform for durability enhancement of molecular catalysts. Chem Mater, 2017, 29: 5720-5726

30 Wang CA, Li YW, Han YF, et al. The "bottom-up" construction of chiral porous organic polymers for heterogeneous asymmetric organocatalysis: MacMillan catalyst built-in nanoporous organic frameworks. Polym Chem, 2017, 8: 5561-5569

31 Zhang X, Kormos A, Zhang J. Self-supported BINOL-derived phosphoric acid based on a chiral carbazolic porous framework. Org Lett, 2017, 19: 6072-6075

32 Lin ZJ, Lü J, Li L, et al. Defect porous organic frameworks (dPOFs) as a platform for chiral organocatalysis. J Catal, 2017, 355: 131138

33 Zou X, Ren H, Zhu G. Topology-directed design of porous organic frameworks and their advanced applications. Chem Commun, 2013, 49: 3925-3936

34 Pei C, Ben T, Qiu S. Great prospects for PAF-1 and its derivatives. Mater Horiz, 2015, 2: 11-21

35 Díaz U, Corma A. Ordered covalent organic frameworks, COFs and PAFs. From preparation to application. Coord Chem Rev, 2016, 311: 85-124

36 Das S, Heasman P, Ben T, et al. Porous organic materials: strategic design and structure-function correlation. Chem Rev, 2017, 117: $1515-1563$

37 Jing LP, Sun JS, Sun F, et al. Porous aromatic framework with mesopores as a platform for a super-efficient heterogeneous Pdbased organometallic catalysis. Chem Sci, 2018, 9: 3523-3530

38 Sun JS, Jing LP, Tian Y, et al. Task-specific design of a hierarchical porous aromatic framework as an ultrastable platform for largesized catalytic active site binding. Chem Commun, 2018, 54: 1603-
1606

39 Ben T, Ren H, Ma S, et al. Targeted synthesis of a porous aromatic framework with high stability and exceptionally high surface area. Angew Chem Int Ed, 2009, 48: 9457-9460

40 Trewin A, Cooper AI. Porous organic polymers: distinction from disorder? Angew Chem Int Ed, 2010, 49: 1533-1535

41 Ben T, Qiu S. Porous aromatic frameworks: Synthesis, structure and functions. CrystEngComm, 2013, 15: 17-26

42 Thomas JMH, Trewin A. Amorphous PAF-1: guiding the rational design of ultraporous materials. J Phys Chem C, 2014, 118: 1971219722

43 Lu W, Yuan D, Sculley J, et al. Sulfonate-grafted porous polymer networks for preferential $\mathrm{CO}_{2}$ adsorption at low pressure. J Am Chem Soc, 2011, 133: 18126-18129

$44 \mathrm{Lu} \mathrm{W}$, Sculley JP, Yuan D, et al. Polyamine-tethered porous polymer networks for carbon dioxide capture from flue gas. Angew Chem Int Ed, 2012, 51: 7480-7484

45 Konstas K, Taylor JW, Thornton AW, et al. Lithiated porous aromatic frameworks with exceptional gas storage capacity. Angew Chem Int Ed, 2012, 51: 6639-6642

46 Garibay SJ, Weston MH, Mondloch JE, et al. Accessing functionalized porous aromatic frameworks (PAFs) through a de novo approach. CrystEngComm, 2013, 15: 1515-1519

$47 \mathrm{Lu} \mathrm{W}$, Verdegaal WM, Yu J, et al. Building multiple adsorption sites in porous polymer networks for carbon capture applications. Energy Environ Sci, 2013, 6: 3559-3564

48 Li B, Zhang Y, Ma D, et al. Mercury nano-trap for effective and efficient removal of mercury(II) from aqueous solution. Nat Commun, 2014, 5: 5537

49 Van Humbeck JF, McDonald TM, Jing X, et al. Ammonia capture in porous organic polymers densely functionalized with brønsted acid groups. J Am Chem Soc, 2014, 136: 2432-2440

50 Yue Y, Zhang C, Tang Q, et al. A poly(acrylonitrile)-functionalized porous aromatic framework synthesized by atom-transfer radical polymerization for the extraction of uranium from seawater. Ind Eng Chem Res, 2015, 55: 4125-4129

51 Hei $\mathrm{ZH}$, Huang MH, Luo $\mathrm{Y}$, et al. A well-defined nitro-functionalized aromatic framework $\left(\mathrm{NO}_{2}\right.$-PAF-1) with high $\mathrm{CO}_{2}$ adsorption: synthesis via the copper-mediated Ullmann homo-coupling polymerization of a nitro-containing monomer. Polym Chem, 2016, 7: 770-774

52 Banerjee D, Elsaidi SK, Aguila B, et al. Removal of pertechnetaterelated oxyanions from solution using functionalized hierarchical porous frameworks. Chem Eur J, 2016, 22: 17581-17584

53 Li B, Zhang Y, Ma D, et al. Creation of a new type of ion exchange material for rapid, high-capacity, reversible and selective ion exchange without swelling and entrainment. Chem Sci, 2016, 7: 2138-2144

54 Lee S, Barin G, Ackerman CM, et al. Copper capture in a thioetherfunctionalized porous polymer applied to the detection of Wilson's disease. J Am Chem Soc, 2016, 138: 7603-7609

$55 \mathrm{Li} \mathrm{B}$, Sun Q, Zhang Y, et al. Functionalized porous aromatic framework for efficient uranium adsorption from aqueous solutions. ACS Appl Mater Interfaces, 2017, 9: 12511-12517

56 Barin G, Peterson GW, Crocellà V, et al. Highly effective ammonia removal in a series of Brønsted acidic porous polymers: investigation of chemical and structural variations. Chem Sci, 2017, 8: 4399-4409

57 Li B, Zhang $\mathrm{Y}$, Krishna R, et al. Introduction of $\pi$-complexation into porous aromatic framework for highly selective adsorption of 
ethylene over ethane. J Am Chem Soc, 2014, 136: 8654-8660

58 Lau CH, Konstas K, Thornton AW, et al. Gas-separation membranes loaded with porous aromatic frameworks that improve with age. Angew Chem Int Ed, 2015, 54: 2669-2673

59 Zhang Y, Li B, Ma S. Dual functionalization of porous aromatic frameworks as a new platform for heterogeneous cascade catalysis. Chem Commun, 2014, 50: 8507-8510

$60 \mathrm{Wu}$ M, Chen G, Liu P, et al. Preparation of porous aromatic framework/ionic liquid hybrid composite coated solid-phase microextraction fibers and their application in the determination of organochlorine pesticides combined with GC-ECD detection. Analyst, 2016, 141: 243-250

61 Comotti A, Bracco S, Mauri M, et al. Confined polymerization in porous organic frameworks with an ultrahigh surface area. Angew Chem Int Ed, 2012, 51: 10136-10140

62 Peng Y, Ben T, Jia Y, et al. Dehydrogenation of ammonia borane confined by low-density porous aromatic framework. J Phys Chem C, 2012, 116: 25694-25700

63 Lau CH, Nguyen PT, Hill MR, et al. Ending aging in super glassy polymer membranes. Angew Chem Int Ed, 2014, 53: 5322-5326

64 Klumpen C, Gödrich S, Papastavrou G, et al. Water mediated proton conduction in a sulfonated microporous organic polymer. Chem Commun, 2017, 53: 7592-7595

65 de Arriba ÁLF, Simón L, Raposo C, et al. Proline imidazolidinones and enamines in Hajos-Wiechert and Wieland-Miescher ketone synthesis. Tetrahedron, 2009, 65: 4841-4845

Acknowledgements This work was supported by the National Basic Research Program of China (2014CB931804) and the National Natural Science Foundation of China (21302061 and 21531003).

Author contributions Chen P and Zhu G conceived the idea, designed and supervised the experiments, and performed the data interpretation. Chen P and Sun JS carried out the experiments. Zhang L and Ma WY helped to carry out the experiments. Chen $\mathrm{P}$ wrote the manuscript. Sun F contributed to the writing of the manuscript. All authors contributed to the general discussion.

Conflict of interest The authors declare no conflict of interest.

Supplementary information Supporting data are available in the online version of the paper.

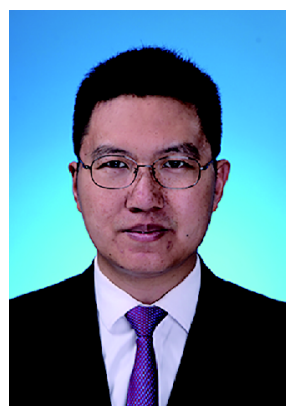

Peng Chen is currently an associate professor at the Institute of Drug Discovery Technology, Ningbo University. He received his $\mathrm{PhD}$ from Lanzhou University in 2011. He worked as an assistant professor in the Department of Chemistry (Jilin University) in 2011-2018. His main research interest includes the synthesis of bioactive compounds, functional organic molecules and functional organic materials.

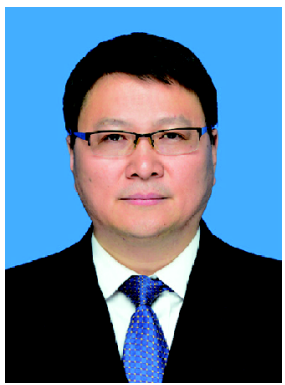

Guangshan Zhu earned his PhD from Jilin University in 1998. He was then immediately appointed as an assistant professor in the Department of Chemistry (Jilin University). In 1999, he worked as a postdoctoral research associate at Tohoku University in Japan. He has been a full professor since 2001, and now holds the Cheung Kong Professorship from the Ministry of Education of China and a Visiting Professorship at Griffith University (Australia). In 2015, he was appointed a full professor at Northeast Normal University. The current research in his group focuses on the design and synthesis of zeolites, metal-organic frameworks, and porous organic frameworks for applications in gas-liquid adsorption, separation, and other advanced applications.

\section{以多孔芳香骨架材料PAF-1作为对映选择性有机催化的超稳定固载平台}

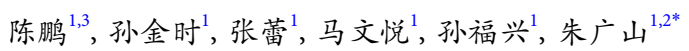

摘要 PAF-1 是最著名的多孔芳香骨架材料(PAF), 它拥有许多优异的性质并且可被用于多个领域. 由高密度的苯环组成的PAF-1材料拥 有刚性结构和亲脂性孔道, 非常适合用作有机催化的平台. 但是迄今为止, 尚未有将其应用到对映选择性有机催化的报道. 本论文以PAF-1 为固载平台, 将手性脯氨酰胺催化位点通过一系列后修饰的方法固载到PAF-1的骨架上, 得到了新颖的手性固载催化剂PAF-1-NHPro. PAF-1-NHPro在催化对硝基苯甲醛和环已酮的Aldol反应的过程中表现出了优良的非对映选择性和对映选择性以及良好的可回收利用 性. 本工作展现了PAF材料在非均相对映选择性有机催化领域的应用前景. 